\title{
Topological Space Partition for Fast Ray Tracing in Architectural Models
}

\author{
Maxime Maria, Sébastien Horna and Lilian Aveneau \\ University of Poitiers, XLIM-SIC, UMR7252 CNRS, Futuroscope Chasseneuil Cedex, Poitiers, France \\ \{maxime.maria, sebastien.horna, lilian.aveneau\}@univ-poitiers.fr
}

Keywords: $\quad$ Interactive Ray Tracing, Beam Tracing, Acceleration Structure, Architecture, Topology, Cells-and-Portals.

\begin{abstract}
Fast ray-tracing requires an efficient acceleration structure. For architectural environment, the most famous is the cells-and-portals one. Many previous works attempt to automatically construct a good cells-and-portals. We propose a new acceleration structure which extends the classical cells-and-portals. It is automatically extracted from the topological model of a given building. It contains a low number of large volumes, all of them linked into a graph model. The scan of our structure is particularly simple and rapid, using all the topological information available from the topological model. The scan can be done for a single ray, or a wide ray packet. We show in this paper that our structure allows an interactive rendering even for large building models, with direct lighting from some thousands of point lights.
\end{abstract}

\section{INTRODUCTION}

Ray-tracing is a rendering technique allowing to compute high-quality realistic images. It allows to render all kinds of visual phenomena such as reflection, refraction, direct or global illumination. Its main disadvantage lies in its high computational cost. Indeed, for each pixel to render, at least one ray is cast through a virtual scene. This simulates the light transport through the scene, and returns the color of the pixel. For a given ray, the problem consists in finding the nearest intersection, among the geometry of the scene. From Whitted's work (Whitted, 1980) to nowadays, many methods have been proposed to improve ray tracing efficiency. Usually, a well-suited acceleration structure is used to reduce the number of useless ray intersection tests.

As part of architectural environments, lighting simulation can be useful, for instance to visualise a building before its construction. General acceleration structures such as kd-trees (Bentley, 1975), BSPtrees (Fuchs et al., 1980), regular grids (Fujimoto et al., 1988) or bounding volume hierarchy (Rubin and Whitted, 1980; Kay and Kajiya, 1986) are not fully adapted to architectural scenes. While they can be used for a broad range of applications, they can produce bad rendering results, particularly with architectural scenes.

Usually, buildings are rendered using a specific acceleration structure, called cells-and-portals. In previous works (Airey et al., 1990; Teller et al., 1994;
Meneveaux et al., 1998; Fradin et al., 2005), the efficiency of cells-and-portals have been demonstrated using only neighbourhood information between volumes.

In this article, we propose a new acceleration structure for ray tracing in architectural environments. This structure consists in a topological model corresponding to a 3 dimensional space partition. It benefits from all topological information available, i.e. neighbourhood relations between volumes (as in previous works), and also between faces, edges and vertices. This new structure is well-suited for ray casting purpose, ray tracing with reflection, refraction and direct or global illumination. It comes with an efficient beam traversal algorithm, that uses all the topological relations to schedule and speed up ray intersection

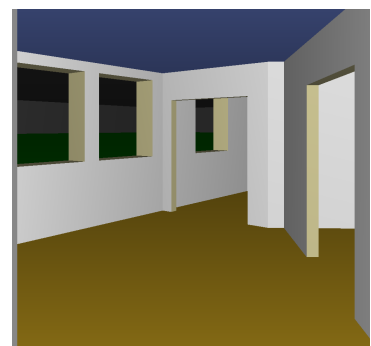

(a) Ray casting, 375 FPS

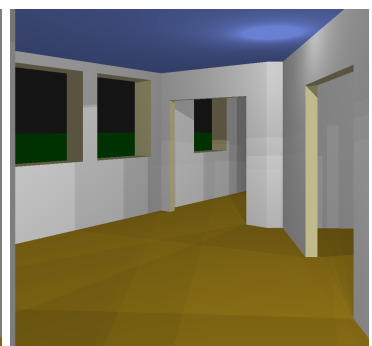

(b) Direct lighting, 22 FPS
Figure 1: Examples of rendering in a building made up of 30k polygons, for $1024 \times 1024$ pixels without anti-aliasing. Left corresponds to a packet ray casting, right illustrates direct lighting from the 199 ponctual light sources in that scene. 
tests. Using this topological information, a simple calculation associates each cell with a list of potentially visible light sources. Thus, our structure traversal leads to interactive frame rates using ray tracing, even for scenes containing many light sources ( $c f$. Figure 1).

This paper is organised as follows: Section 2 reviews previous works on acceleration structure for ray tracing in general and then specifically focuses on those dedicated to architectural environments. Section 3 introduces our topological acceleration structure. Section 4 presents our topological strategy used to accelerate the traversal of one ray through the scene. Section 5 extends the traversal of our structure for reflection and lighting, as examples of its capabilities. Section 6 gives and analyses some ray-tracing results. At last, Section 7 concludes and gives some future work lines.

\section{RELATED WORKS}

Since 1968 and Appel's works (Appel, 1968), ray tracing has been used to compute high quality realistic images (Whitted, 1980; Cook et al., 1984; Glassner, 1989) including reflection and refraction phenomena or global illumination account. While the first interactive ray tracer was proposed by Parker et al. in (Parker et al., 1999) using a large shared memory supercomputer, interactive frame rates rendering using ray tracing still remains a non trivial task. Most of the time, an acceleration structure is built over the scene in order to improve ray tracing performances. In this section, we briefly present the common acceleration structures. Then, we focus on structures dedicated to the architectural environments.

\subsection{General Acceleration Structures}

A naive approach of tracing a ray through a scene would consist in testing the intersection between rays and all the polygons making up the scene. For interactive rendering, such an approach is obviously prohibited. An acceleration structure is designed to decrease this linear complexity. It relies on a special organisation of the geometry which aims to speed up a random access to a polygon to a logarithmic time. As a consequence, the number of ray intersection tests performed is highly reduced and the rendering is faster. Many acceleration structures have been proposed in the literature such as kd-trees (Bentley, 1975), bounding volume hierarchies (BVH) (Rubin and Whitted, 1980; Kay and Kajiya, 1986) or regular grids (Fujimoto et al., 1988). A survey comparing all these ac- celeration structures on CPU can be found in (Havran, 2000)

These structures are general, in that they work with any kind of scenes. Nevertheless, in some cases their traversal cost can become very high. That is the case for architectural environments. First, buildings can contain little but highly detailed objects inside big empty spaces. This kind of configuration is well known as the "teapot in the stadium" problem. It slows down traversal performances of regular structures like regular grids. A second problem appears with architectural environments with walls in general configurations. With axis-aligned partitioning methods, such as kd-tree or BVH, the cutting splits do not respect the wall positions and produce a complex subdivision scheme. These two problems explain for the most part why general acceleration structures are not favoured to render architectural scenes.

\subsection{Architectural Acceleration Structures}

Architectural scenes are made up of large occlusive surfaces such as floors, ceilings or walls. From a given point of view only a small part of the scene is visible. The structure called cells-and-portals takes advantage of this particular structural organisation.

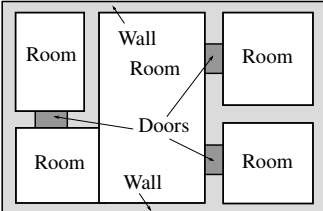

(a)

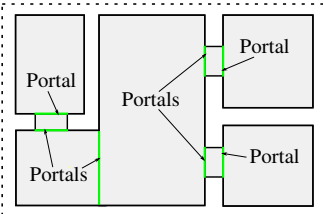

(c)

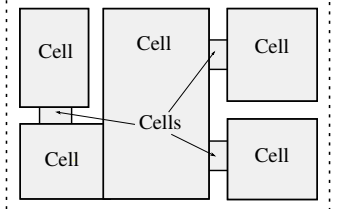

(b)

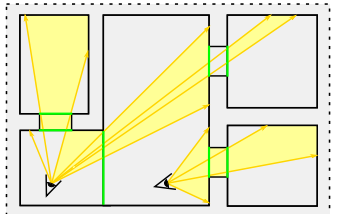

(d)
Figure 2: 2D cells and portals representation and usage: (a) building representation; (b) rooms and doors correspond to cells; (c) portals are shared by 2 cells; (d) rays hitting a portal are propagated through neighbouring cells.

In such a structure, a cell represents a volume in which light can be propagated, such as a room or an opening. A portal corresponds to a face incident to two cells ( $c f$. Figure 2). A cells-and-portals is stored in a graph, where a node represents a cell and an edge corresponds to a portal. The principle of traversal consists in scanning such a graph. From a given cell $C$, a ray $r$ can exist only by a face incident to $C$. If this 
outgoing face is a portal, then $r$ is propagated in the neighbouring cell; else it is stopped ( $c f$. Figure 2(d)). Due to the local complexity of this process, it remains efficient even for large architectural scenes.

Several methods have been proposed to construct such a structure starting from a list of polygons. Airey et al. (Airey et al., 1990) described a method for architectural scenes with axis-aligned walls. The subdivision is performed using a kd-tree. It has been extended for general convex cells (non necessarily axis-aligned) using a BSP-tree by Teller et al. (Teller and Séquin, 1991). Meneveaux et al. (Meneveaux et al., 1998) used a rule-based system which consists in finding the openings (i.e. the portals), in order to use them to construct cells that fit better with walls. All these works aim at extracting neighbourhood information between volumes. The problem of these methods is that the cells are not optimally determined so that a room could be uselessly split several times.

In (Fradin et al., 2005), cells-and-portals are directly extracted from architectural environments which are built by using a dedicated topological modeller. Resulting scenes are adapted for lighting simulation in large buildings as shown in (Fradin et al., 2005; Fradin et al., 2006). Neighbourhood information between volumes are directly known from the topology. This work produces an optimal cells-andportals structure in which a room is represented by an unique cell. Nevertheless, it has two main drawbacks. Firstly, the full potential of the topological model is not exploited: Indeed, the neighbourhood relations between faces, edges and vertices are known but unused. Secondly, the construction is entirely manual so that designing a scene is a long and tedious task. In addition, scenes can not be validated when no architectural constraints or editing operations are provided.

In this paper, we use the model introduced by Horna et al. in (Horna et al., 2009). It is also a topological model, but it can produce automatically the piece of information needed to cell-and-portals traversal. This model is described in more details in Section 3. The associated modeller allows to automatically generate the 3 dimensional structure from an architectural plan (Horna et al., 2007). Moreover, contrary to Fradin et al. we propose to exploit all its topological properties, leading to a very efficient traversal.

\section{TOPOLOGICAL STRUCTURE}

In this section, we present our topological acceleration structure for architectural environments.

\subsection{Generalized Maps}

An architectural environment can be considered as a set of volumes. Each volume corresponds to a unique element in the scene (room, wall, door, window, etc.). Therefore, an adjacency graph is sufficient to store such a structure.

Our acceleration structure is based on the topological model introduced by Horna et al. (Horna et al., 2009). It uses the notion of topological dimension, which is associated with geometrical objects: Dimension 3 with a volume, dimension 2 with a face, dimension 1 with an edge and dimension 0 with a vertex. This model contains topological relations between all these geometric elements, from dimension 0 to 3. It is based on generalized maps, or G-maps (Lienhardt, 1994), extended by the formal definition of a set of topological, geometrical and semantic constraints specifically dedicated to architectural modelling. These constraints ensure the model validity during all the modelling process.

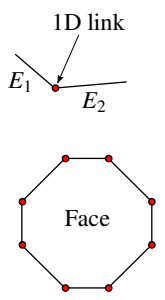

(a)

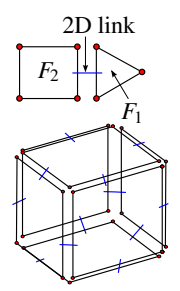

(b)

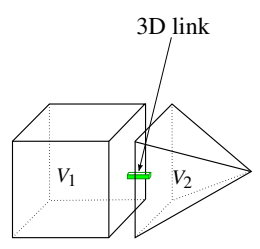

(c)
Figure 3: Topological links in each dimension: (a) links of dimension 1 bind edges to make faces; (b) links of dimension 2 tie together faces to generate volumes; (c) volumes are tied with links of dimension 3.

In an architectural environment, all elements are real world objects. In such a scene, two elements can not occupy the same space, and the whole space is filled, authorizing a space partition. Consequently, topologically speaking, a building should be defined as a subdivision of space into volumes, faces, edges and vertices. In this article, we only recall the main principles of generalized maps applied to buildings, i.e. the neighbourhood information. All the definitions and properties of n-dimensional generalized maps can be found in (Lienhardt, 1994). The Figure 3 shows how topological information is organised (for simplification, links of dimension 0, binding two vertices to make an edge, are not represented).

\subsection{Architectural Topological Model}

Architectural scenes are represented by a 3 dimensional oriented and closed space subdivision composed of elements with a significant thickness. Each 


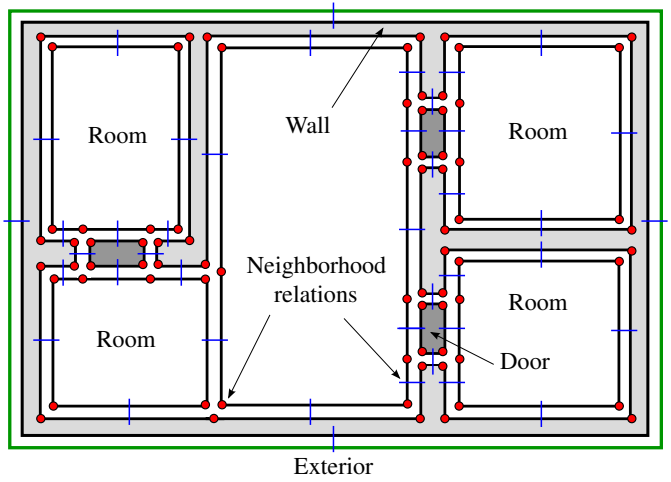

(a)

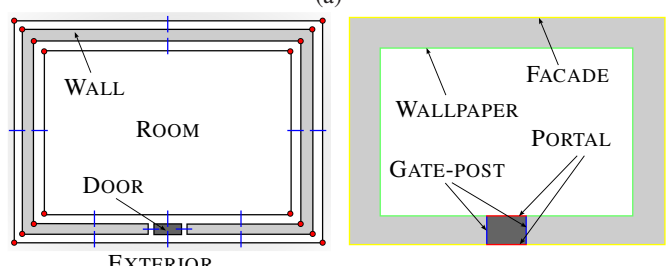

(b)

Figure 4: Topology and semantic: (a) 2 dimensional scene complying the topological and semantic properties; each face (in 3D, each volume) corresponds to a unique element (room, wall, etc.); topology represents neighbouring relations. (b) An edge (in 3D, a face) incident to two cells becomes a PORTAL while a non-portal edge has a specific semantics (WALLPAPER, FACADE, GATE-POST).

volume is identified by a unique semantic according to its nature: ROOM, DOOR, WALL, GROUND, CEILING or EXTERIOR. The Figure 4(a) illustrates a scene complying to this property. Note that all descriptions made in dimension 2 are extensible to dimension 3, since G-maps are homogeneous in all dimensions.

From this model, we design a cells-and-portals structure in the following way: In the cells-andportals philosophy, a cell is a volume where light can be propagated. This corresponds to a given subset of semantic: ROOM, DOOR, EXTERIOR. Then, with our architectural model, the cells can be deduced automatically from the volumes.

In the same manner, a portal is a face which can be crossed by the light. With our model, portals are automatically deduced from semantics. Indeed, the only way to stop the light is to find a non cell volume. Then, using the semantic information stored into our structure, a face is a portal if and only if it links two cells ( $c f$. Figure 4(b)).

In fact, our model is also used to find automatically the semantics of each face, even the non portal one. This allows to deduce the reflecting properties of non portal faces, and is exploited during the rendering. For example, a face incident to a RoOM and a WALL is identified as a WALLPAPER, while one inci- dent to a DOOR and a WALL becomes a GATE-POST.

\subsection{Our Acceleration Structure}

Architectural environments are mostly composed of rooms made with large vertical planes for the walls, and horizontal planes for floor and ceiling. Hence, we represent each volume by two horizontal faces (upper and lower ones) and a set of vertical bounding faces.

Each vertical face can be divided into several vertical parts, eg. to represent a door or a window ( $c f$. Figure 5).

Our structure results in a space partition, representing a cells-and-portals structure in which a cell corresponds to an unique room. Nevertheless, for rendering efficiency our structure traversal is optimised for convex cells. Then, each concave cell is automatically subdivided into a set of convex cells (sharing portals). Thus, finally, a room is made of several cells.

Our acceleration structure is stored into a compact graph structure. A node corresponds to a cell and the graph links are the topological links of dimension 3 (the neighbourhood between cells i.e. the portals). Each node (so cell) contains its 2 dimensional lower face made of vertices. It is ordered counter clockwise (i.e. from right to left from inside the volume). Each vertex contains a list of horizontal edges representing the vertical faces, with their semantics. It is ordered from the bottom up. At least, a node contains a ceiling height. This particular data structure (organised according to topological elements) allows to schedule and then to speed up the traversal process. Next section describes the traversal algorithm for one ray.

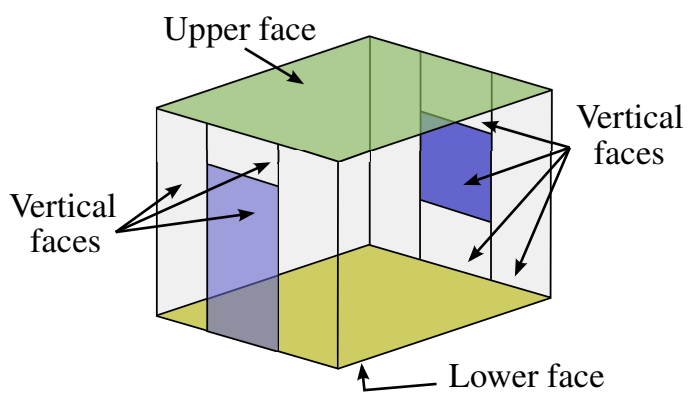

Figure 5: Volume characteristics in building: They are composed of 2 horizontal faces and several vertical faces corresponding to volume border.

\section{STRUCTURE TRAVERSAL}

The traversal of our structure requires displacement in a graph. In fact, it consists in a classical cells- 
and-portals traversal but improved by using available topological links in all dimensions.

In this section, we firstly present the principle of a basic ray casting in our topological structure. Then, we recall the Plücker coordinates, which are used for intersection tests. Finally, we explain our topological strategy use to improve the outgoing face search.

\subsection{Basic Ray Casting}

Without considering the optimisation brought by our topological strategy for the outgoing face search, our acceleration structure is a kind of cells-and-portals. Then, a basic ray casting is a straightforward problem. All the rays are propagated through the scene starting from the volume containing the camera. Nevertheless, this algorithm hides two key points.

The first on resides in the determination of the initial volume (or camera volume). This volume is calculated once and only once. Then, when the camera is moved by the user, the camera volume is updated according to the translation vector defining the movement; this costs nothing since it simply consists in casting a finite and short ray. Thus, the updated camera volume corresponds to the ray arrival volume.

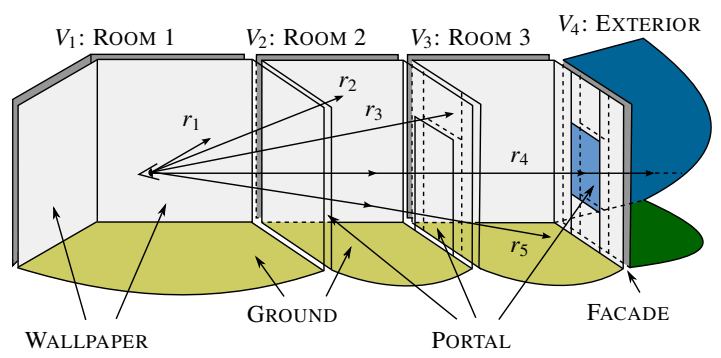

Figure 6: Scene traversal: Rays start from volume $V_{1}$ and are propagated when they hit a face PORTAL, or are stopped when they hit a boundary face (WALLPAPER, GROUND, etc.). As an example, ray $r_{1}$ hits a face in $V_{1}$ with semantics WALLPAPER, while ray $r_{4}$ goes through three portals before being propagated inside volume $V_{4}$ of semantics EXTERIOR.

The second key point lies in finding the outgoing face of a given ray in a given volume. If this face has a semantics PORTAL, then the ray is propagated through its neighbouring volume by using topological relation of dimension 3. Else, the hit face is a boundary face (semantics WALLPAPER, GROUND, etc.) and the ray is stopped ( $c f$. Figure 6).

As mentioned in Section 2.2, the global number of faces making up the scene does not really matter due to the local complexity of the traversal. Given that the propagation of a ray from a volume to its neighbour is constant by using topological relation of dimension 3 (i.e. volume neighbouring), the efficiency of such an algorithm lies in finding quickly the outgoing face of a given ray inside a volume. To improve that and thus the global traversal, we propose an outgoing face search scheduling based on the topology of the scene. While previous works only used neighbouring relation between volumes (of dimension 3 ), we also use relation between faces (dimension 2), edges (dimension 1) and vertexes (dimension 0), in order to speed up the outgoing face search. Section 4.3 explains in details this process.

\subsection{Plücker Coordinates}

Plücker coordinates was firstly introduced in (Plücker, 1865). Generally speaking, they are used to represent linear subspaces of dimension $k$ in a projective space of dimension $n$. In computer graphics, they represent oriented lines of 3 dimensional geometrical space. They are particular point in the projective 5 dimensional space $\mathbb{P}^{5}$. More precisely, an oriented line $l$ defined by two distinct points $A$ and $B$ is represented by a sextuplet of coordinates $\Pi_{l}=\{u=b-a: v=a \times b\}$, where the 3 dimensional vectors $a$ and $b$ are respectively the position of the points $A$ and $B$. Hence, the vector $u$ corresponds to the direction of the line, while $v$ is its position (or its mechanical moment).

As a example of Plücker coordinates usefulness, they are used in visibility problems as shown in (Teller and Hanrahan, 1993; Charneau et al., 2007; Fang, 2010; Mora et al., 2012). Here, we use them to determinate the relative orientation between two oriented lines via the operator side (Shoemake, 1998). This operator is defined for two lines $l$ and $l^{\prime}$ as side $\left(\Pi_{l}, \Pi_{l^{\prime}}\right)=u . v^{\prime}+u^{\prime} . v$. If the result is negative, $l$ turns clockwise around $l^{\prime}$; if it is positive, $l$ turns in the counterclockwise around $l^{\prime}$; else, when side is null, the two lines have a common point (in fact they are coplanar, and so they meet at least at infinity).

\subsection{Outgoing Face Search}

Using the topological relations available in our structure, we develop a simple and efficient algorithm for searching the outgoing face of a given ray into a volume ( $c f$. Algorithm 1). It is divided into two main steps.

Let us recall that each volume is convex. Then a ray can only exit through an unique face. First, we consider the volume as an infinite vertical polyhedron, ignoring the upper and lower faces. Therefore, we start to search the outgoing infinite vertical section (Algorithm 1 - line 3-7).

An infinite vertical section is bounded by two vertical lines oriented from the bottom up. Using Plücker 
lines, it is trivial to test if a ray exits through a given vertical section, by testing its side product with the two bounding lines. Indeed, a ray intersects such a section when it goes through the left (resp. right) of the right (resp. left) line. This search is done linearly, by scanning the vertices of the lower face. It uses both topological relations of dimension 1 and 0 (i.e. links between edges and vertices). Considering the low number of vertical sections per volume (in average 4.37) a more complex scan (like dichotomy) is useless.

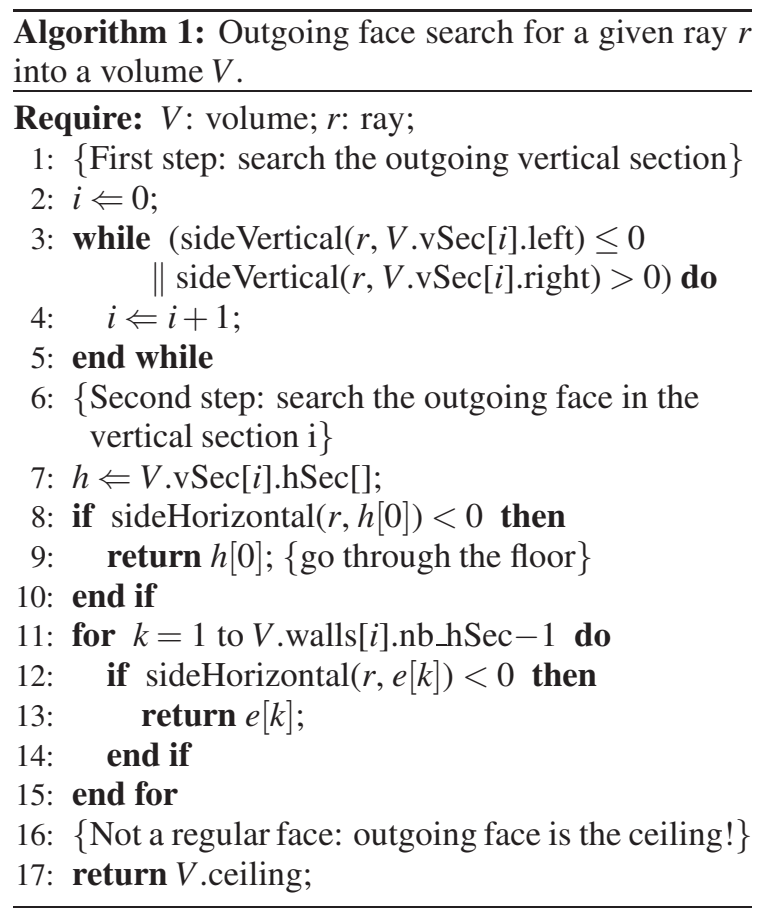

It should be noticed that this first step is done strictly in dimension 2 (see Figure 7). If $x$ and $y$ are the 2D coordinates of a line bounding the wall (with direction $u=z$ ), and for a ray defined with Plücker coordinates $\Pi_{l}=\left\{\pi_{0}, \pi_{1}, \pi_{2}, \pi_{3}, \pi_{4}, \pi_{5}\right\}$, then the side operator is reduced to: $y \times \pi_{0}-x \times \pi_{1}+\pi_{5}$.

Once the outgoing infinite vertical section is found, we search for the outgoing face. We consider the outgoing vertical section as a set of infinite faces bounded by two horizontal lines (see Figure 8 ). Let us recall that these lines are oriented from right to left in our acceleration structure. Then we can use Plücker side product to test the ray intersection. We start from the lower edge (Algorithm 1 - line 9-12): If the result is negative, the ray turns clockwise around the edge (the ray goes below the edge); then the floor is hit and can be returned. Otherwise, we repeat this process with upper edges by scanning them (by using topological relations of dimension 2, i.e. links between

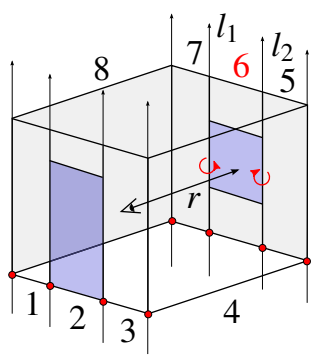

(a)

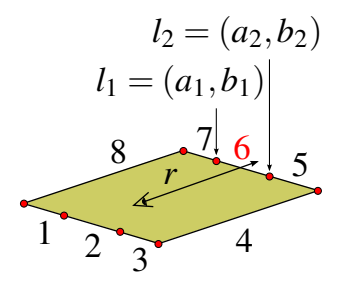

(b)
Figure 7: First step of outgoing face search: (a) the cell is considered as an infinite vertical polyhedron; a vertical section is bounded by two lines oriented from the bottom up; outgoing vertical section is 6; (b) the process is strictly done in $2 \mathrm{D}$.

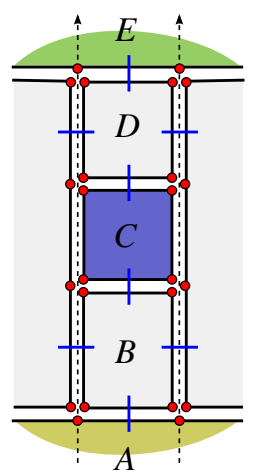

(a)

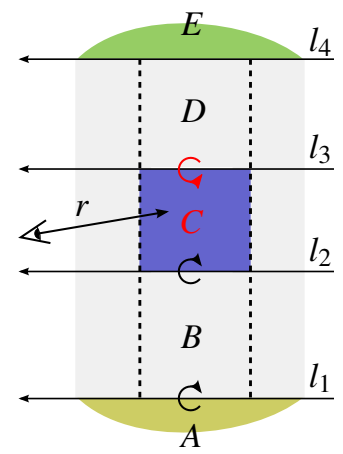

(b)
Figure 8: Second step of outgoing face search: (a) a vertical section is divided into several faces, including ground and ceiling; (b) a face is bounded by two horizontal lines oriented from right to left; outgoing face is $C$.

faces) until finding the outgoing face (Algorithm 1 line 13-17). If none face is found, then the ceiling is returned (Algorithm 1 - line 18). Once more, a nonlinear strategy is useless in front of the low number of faces (in average 2.11). Considering that bounding lines are only horizontal, Plücker side operator is reduced to four products and five additions.

To sum up, outgoing face search is optimised by using topological relations of all dimensions:

- Dimensions 0 (vertex) and 1 (edge) to scan the lower face of a volume and to find the outgoing vertical section.

- Dimension 2 (face) to change of face within a vertical section and determine the outgoing face.

- Dimension 3 (volume) to move from a volume to its neighbour when propagating a ray. 


\section{EXTENDED USES}

This section discusses about the integration of our model into a ray tracing application to render architectural scenes, using wide ray packets and secondary rays (reflection, refraction, and light rays).

\subsection{Ray Packet}

The basic ray casting algorithm is modified in order to group the rays in packets. In practice, we use can very wide packet: depending on the number $t_{h}$ of threads, we divide the image into $2 \times t_{h}$ packets. In our experiments, this leads to 16 packets for $1024 \times 1024$ pixels.

A packet is defined by its 4 corners, corresponding to 4 pixels in the ray casting (and idem for secondary rays). From the initial volume, the outgoing face of this 4 rays is searched according to Algorithm 1. When the results are coherent (the 4 rays goes through the same face), the algorithm computes successfully the outgoing face for all internal rays.

Else, when rays are incoherent, the algorithm can not continue for the given packet. For each corner rays, all the current data is stored: i.e. the identifiers of the volume, of the vertical section and of the face of this vertical section. Then, the packet is split in 2 or 4 parts, depending on the incoherence and each part continue in the same way, from the same initial position.

In practice, this strategy is very efficient. It uses SIMD float point instruction as, for instance, in the kd-tree packet traversal proposed in (Wald et al., 2001). Since the new packets continue from where they are split we do not spend time in a new structure scanning.

\subsection{Reflection and Refraction}

Our model allows to associate any kind of behaviour to a face thanks to the face semantics. This allows to add some reflection or refraction properties to the surfaces, as an example for panes.

When a ray hit a reflective or refractive surface, its trajectory is modified. For a both reflective and refractive surface, it is also subdivided. Since our traversal algorithm have a local scope, the cost of a reflective or refractive ray stays about the same that for primary rays. For example, with a specular surface, a ray can be reflected a given number of times. For each bounce, a new ray have to be cast, with new Plücker coordinates. In fact, specularity account is not a difficult task. The new coordinates of the specular ray are computed according to a traditional scheme ( $c f$. Section 4.2) and the ray is propagated by using the Algorithm 1, or its packet version. The main difference resides in the starting cell: The volume containing the reflection point is not the same that the camera one. Fortunately, this volume do not have to be computed: It corresponds to the arrival volume of the primary ray.

\subsection{Direct Illumination}

Computing the direct illumination is a good way to evaluate the robustness of our structure in case of spatially incoherent rays. That implies new rays (light rays) to be cast through the scene, to determine the point-to-point visibility between the primary ray intersections and the point light sources. Each new ray is simply cast, as a primary ray (see Section 4). Then, lighting is computed according to the visibility and the chromatic intensity of each visible source.

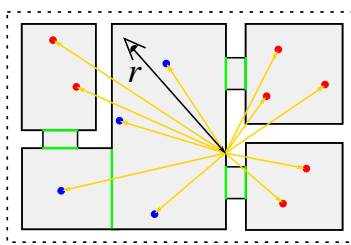

(a)

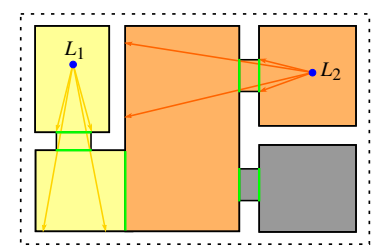

(b)
Figure 9: Direct illumination: (a) for the ray $r, 11$ light rays are launched while only 4 are visible (in blue); (b) cells crossed by light beams are potentially illuminated, allowing to decrease the number of light rays.

The main difficulty for direct illumination account in architectural environments lies in the huge number of light sources which highly increase the number of rays to be cast, leading to low frame rates. Fortunately, given that buildings are composed of large occlusive surfaces, too many useless tests are performed ( $c f$. Figure 9(a)). Cells-and-portals structures are used in (Airey et al., 1990), (Teller and Séquin, 1991), (Luebke and Georges, 1995) or (Meneveaux et al., 1998) to compute the potentially visible set (or PVS) in architectural environments. From the same perspective and for efficiency purpose, we choose to compute the set of potential visible lights for each cell making up our scenes. We proceed by launching continuous beams from a light source, one through each opening of the volume containing the light source ( $c f$. Figure 9(b)). Parts of the beam which hit a face PORTAL are recursively propagated through neighbouring cells until being stopped by a non-portal face. As usual, the propagation is performed by using topological relation of dimension 3 . Each cell crossed by the beam is potentially illuminated by the light source. 
Table 1: Characteristics of our test scenes: number of rooms, cells, polygons, lights and memory size in megabytes.

\begin{tabular}{|l|c|c|c|c|c|}
\hline Scenes & \# rooms & \# cells & \# polygons & \# lights & memory (Mb) \\
\hline \hline HOUSE & 8 & 509 & 1.166 & 8 & 0.46 \\
BUILD1 & 46 & 2.333 & 8.557 & 55 & 2.03 \\
BUILD2 & 135 & 8.258 & 29.937 & 199 & 6.82 \\
BuILD3 & 1755 & 95.932 & 369.521 & 2557 & 80.13 \\
\hline
\end{tabular}

Table 2: Results: for each scene we give the average number of frame calculated per second (FPS), and the average number of rays cast per second in millions (Mrays/s).

\begin{tabular}{|l||cc|cc|cc|cc|}
\hline Scenes & \multicolumn{2}{|c|}{ HOUSE } & \multicolumn{2}{c|}{ BUILD1 } & \multicolumn{2}{c|}{ BUILD2 } & \multicolumn{2}{c|}{ BUILD3 } \\
\hline Results & FPS & Mrays/s & FPS & Mrays/s & FPS & Mrays/s & FPS & Mrays/s \\
\hline \hline Ray casting & 65.09 & 68.26 & 51.28 & 53.78 & 51.35 & 53.85 & 46.86 & 49.14 \\
Packet casting & 388.16 & 407.01 & 384.13 & 370.68 & 375.13 & 393.35 & 341.66 & 358.25 \\
Packet Lighting & 128.71 & 419.75 & 66.64 & 367.05 & 60.48 & 352.09 & 34.49 & 246.91 \\
\hline
\end{tabular}

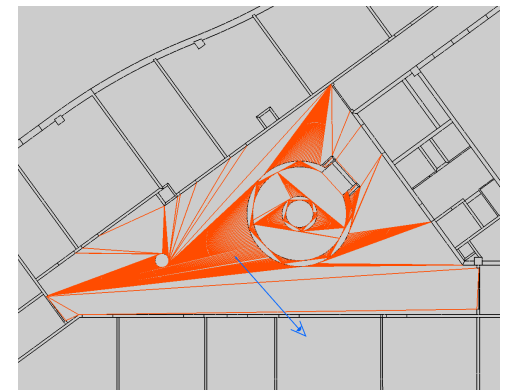

(a) Convexification using (Fernández et al, 2008)'s method; Rays cross 9.139 .670 volumes in all to render the image (c).

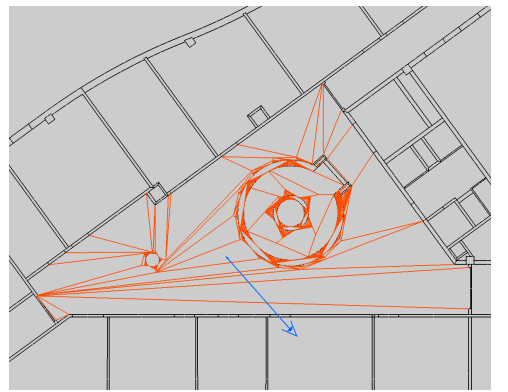

(b) Convexification extended with bounding boxes and topology; Rays cross

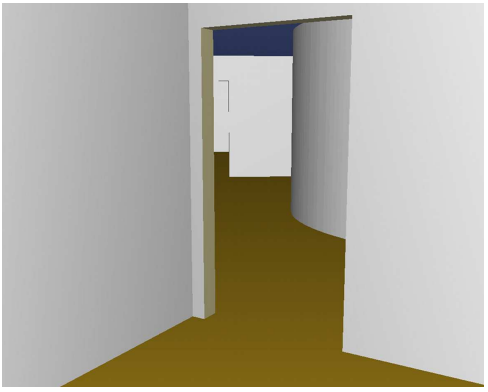

(c) Associated rendered image; 314,31 FPS with convexification (a) and 377,28 FPS with (b). to render the image (c).

Figure 10: Comparison of the convexification of a room.

Instead of having to cast a light ray for each light source, computing PVS allows to highly reduce the number of lights to a local list of potentially visible sources. As an example, in one of our test scene which contains 199 light sources, the average number of light rays per image is reduced from 208.67 millions to 10.14 millions (-95.14\%).

\section{RESULTS}

We evaluate the performances of our acceleration structure on a Intel ${ }^{\circledR}$ Core $^{\mathrm{TM}}$ i7 CPU $960 @ 3.20 \mathrm{GHz}$ with $12 \mathrm{~GB}$ of RAM. The traversal algorithm runs in parallel using OpenMP. The packet version also uses 4-wide SIMD floating point instructions (Intel ${ }^{\circledR}$ SSE). Performances are given in terms of:

- FPS: frames calculated per second.

- Mrays/s: millions of rays cast per second.

The rendering is done for image of $1024 \times 1024$ pixels with one ray per pixel (without anti-aliasing). In order to have relevant statistics, we use a way-point system to keep the same points of view for each algorithm tested. These points of view result from a walk- through into each scene; then the number of points of view can differ according to the size of the scene.

Notice that the rendering time include the shading, using a Lambertian $b r d f$. With ray-casting, a virtual point light is positioned at the camera location, and used for the calculation of the cosine of the incident direction with respect to the surface normal.

\subsection{Scenes}

We present four of our test scenes which are more or less complex in terms of size, number of polygons and light sources. Their main characteristics are summarised into Table 1. The smallest scene corresponds to a house made up of 8 rooms ( $c f$. Figure 12). It has 1.166 polygons and contains 8 light sources. The scenes BUILD1 and BUILD2 represent two distinct floors of a large administration building ( $c f$. Figures 13, 1 and 11). They are respectively made up of 46 and 135 rooms, and contains 8.557 and 29.937 polygons with 55 and 199 light sources. The last scene, BUILD3, is a very large building made up of 13 floors with 1755 rooms, with 369 thousands of polygons and 2557 point light sources ( $c f$. Figure 14). These four scenes result directly from our topological modeller. 


\subsection{Results and Discussion}

\subsubsection{Primary Ray Casting}

We propose two different algorithms for primary ray casting. While the first one works pixel per pixel, the second corresponds to the packet traversal described in Section 5.1. These two algorithms have been evaluated with each of our test scenes.

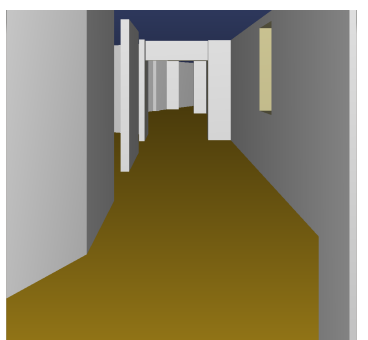

(a) Ray casting (48 FPS).

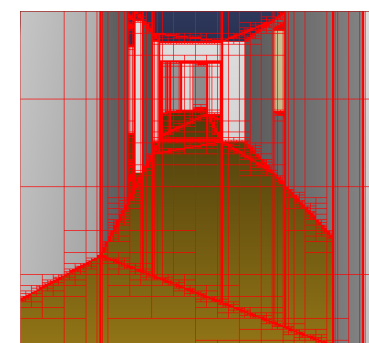

(b) Ray packets (356 FPS).
Figure 11: Illustration of primary ray casing with scene BUILD2.

We can notice that primary ray casting using our topological structure is very efficient (see Table 2). The Figure 11(a) shows the rendering for the second building test scene, using a simple ray casting. The Figure 11(b) proposes the same point of view, but using and emphasizing ray packets. Clearly, the packet are split only near cell boundaries. For large wall, ground or ceiling parts, a wide ray packet can be efficiently traced with our acceleration structure.

Table 3 compares the performances of these two methods with those measured by using the ray tracing kernel Embree (Ernst and Woop, 2011). We can observe both of them are faster than Embree. Our ray casting method is more than 4 times faster and the packet version is more than 30 times faster.

Table 3: Comparison with Embree ray tracing software (Ernst and Woop, 2011).

\begin{tabular}{|l|c|c|c|}
\hline Scenes & Embree & Ray casting & Packet casting \\
\hline \hline HOUSE & 18 & 65.09 & 388.16 \\
BUILD1 & 12 & 51.28 & 384.13 \\
BUILD2 & 10 & 51.35 & 375.13 \\
BUILD3 & 8 & 46.86 & 358.25 \\
\hline
\end{tabular}

Since our algorithm have a local complexity, the size of the scene does not matter since the data could fit easily on the available memory. This complexity takes into account the number of volumes crossed by the ray and the number of polygons making up these volumes (which impact on the outgoing face search). Indeed, performances are reduced in case of a cell split a lot of times or in case of volumes containing too many polygons (i.e. not enough split). Thus, the scene must be convexified using a well suited strategy.

Analogous to the well-known surface area heuristic (SAH) (Goldsmith and Salmon, 1987; MacDonald and Booth, 1990), a concave cell have to be split considering its area and the expected cost of tracing an infinite random ray through that cell. In fact, we search for a split scheme which have the lowest SAH cost possible. The main problem with convexification occurs in case of curved walls as illustrated on Figure 10. The Figure 10(a) shows a room convexified with the method presented in (Fernández et al., 2008). Because of curved walls, many splits are performed, creating a dense partition. Then, rendering an image requires to cross a lot of volumes so that performances are highly reduced. Our method is illustrated in Figure 10(b): Convexification is extended by constructing a bounding box around curved walls to restraint all the splits in a small space, leading to a better SAH cost. The use of such a strategy offers a good compromise and leads to better performances. Notice that convexification process is automatically performed with our modeller and takes from seconds to few minutes according to the size of the scene.

\subsubsection{Direct Lighting}

Taking into account direct lighting means casting a light ray for each light source. For one light source this should twice the number of rays. Then, for $x$ light sources this can dramatically reduce the number of frames per second. As explained in Section 5.3, we compute the PVS for each cell in our structure to reduce the number of light rays.

Table 2 shows that direct lighting makes the number of rays per second drop linearly. Nevertheless, this reduction is not linear with respect to the number of light sources. Some rendering examples are proposed in the Figures 1, 12, 13 and 14.

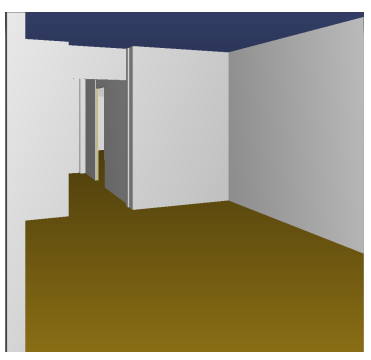

(a) 371 FPS.

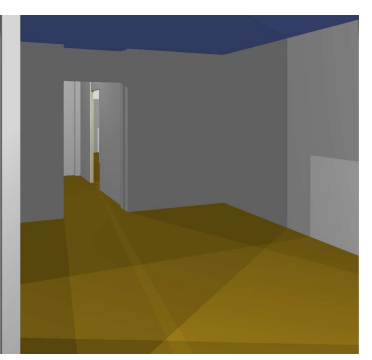

(b) 75 FPS.
Figure 12: HousE without (a) or with (b) lighting from 8 light sources.

This fall in terms of FPS is brought by two causes. First, new Plücker coordinates have to be computed 


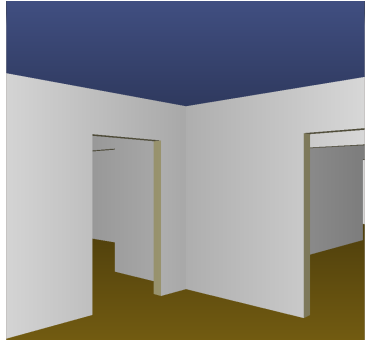

(a) 347 FPS

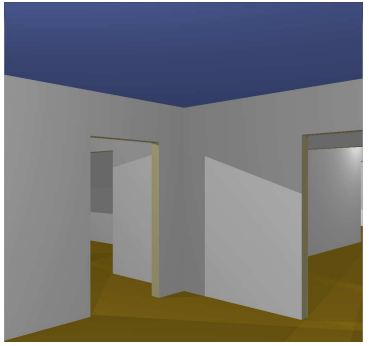

(b) 53 FPS

Figure 13: BUILD1 without (a) or with (b) direct lighting. The scene contains 55 light sources.

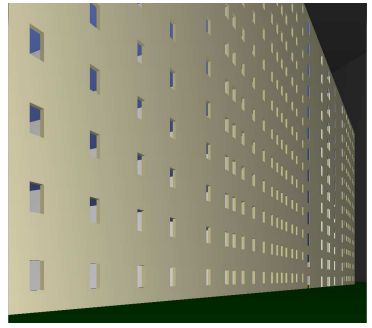

(a) 155 FPS

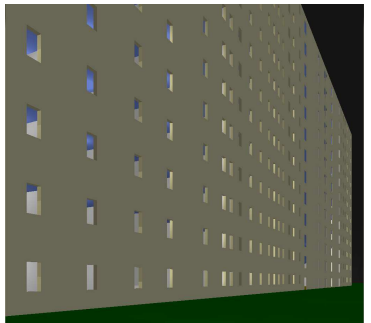

(b) 0.24 FPS.
Figure 14: BUILD3 without (a) or with (b) direct lighting from 2557 lights. Most of the lights are visible outside, leading to a small FPS.

for each light ray to be cast. Secondly, it is due to spatial ray incoherence which leads to incoherent memory accesses. The robustness of our topological structure in terms of number of rays cast per second allows to think that it could be used efficiently to compute global illumination in architectural environments.

\section{CONCLUSIONS}

This article proposes a new acceleration structure for ray tracing dedicated to architectural environments. It is generated automatically from a topological modeller, without human interaction. It takes advantage of the architectural building constraint: it corresponds to a 3 dimensional oriented and closed space subdivision composed of elements with a significant thickness.

Our structure is a kind of advanced cells-andportals, with two main improvements: Firstly, thanks to our topological model, cells-and-portals cutting planes fit perfectly to the building topology. Secondly, while previous cells-and-portals traversal algorithms used only neighbouring relations between volumes, we propose to take advantage of all the topological information available, to schedule the outgoing face search, and to accelerate the whole process.

The traversal of our acceleration structure is implemented on CPU, both with single rays or with ray packets. Our experimentation shows that our structure traversal is very efficient. Our rendering tool computes hundreds of millions of rays per second, using only a single 4-core processor. Consequently, it allows to render images interactively, for ray casting or ray tracing, with direct lighting or not.

We think that our structure and its traversal algorithm can run onto a GPU, taking advantage of the high number of cores. It should be interesting to study the results of such a structure on GPU, especially when a large number of light sources are used.

We also think that our structure can be used to include piece of furniture quite easily. Indeed, using bounding boxes surrounding high detailed objects, we should be able to include them into the topological model. During the rendering step, the rendering could be done by combining our structure with a more classical one. Thus, our topological structure traversal performances would highly depend on the acceleration structure used to render the object.

Then, a logical next step will be to adapt our topological partition to general objects in order to include it directly in our architectural structure.

The main disadvantage of our model relies on the topological modeller. It could not be generated with only a geometric description of scene, without any topological information. As a future work, we plan to adapt our ideas to general objects described only by their geometry. We aim to use generalized maps to connect geometric elements themselves, in order to generate automatically a topological acceleration structure from scratch, for architectural scenes or not. This step would allow to directly include furniture in our architectural structure and thus to avoid the dependence on an auxiliary acceleration structure.

\section{ACKNOWLEDGEMENTS}

Authors thanks the Région Poitou-Charentes for their funding support.

\section{REFERENCES}

Airey, J. M., Rohlf, J. H., and Brooks, Jr., F. P. (1990). Towards image realism with interactive update rates in complex virtual building environments. SIGGRAPH Comput. Graph., 24(2):41-50.

Appel, A. (1968). Some techniques for shading machine renderings of solids. In Proceedings of the April 30-May 2, 1968, Spring Joint Computer Conference, AFIPS '68 (Spring), pages 37-45. ACM. 
Bentley, J. L. (1975). Multidimensional binary search trees used for associative searching. Commun. ACM, 18(9):509-517.

Charneau, S., Aveneau, L., and Fuchs, L. (2007). Exact, robust and efficient full visibility computation in the Plücker space. Visual Computer, 23(9-11):773-782.

Cook, R. L., Porter, T., and Carpenter, L. (1984). Distributed ray tracing. SIGGRAPH Comput. Graph., 18(3):137-145.

Ernst, M. and Woop, S. (2011). Embree: Photo-realistic ray tracing kernels.

Fang, Q. (2010). Mesh-based Monte Carlo method using fast ray-tracing in Plücker coordinates. Biomed. Opt. Express, 1(1):165-175.

Fernández, J., Tóth, B., Cánovas, L., and Pelegrín, B. (2008). A practical algorithm for decomposing polygonal domains into convex polygons by diagonals. TOP , 16(2):367-387.

Fradin, D., Meneveaux, D., and Horna, S. (2005). Out-ofcore photon-mapping for large buldings. In Proceedings of Eurographics symposium on Rendering.

Fradin, D., Meneveaux, D., and Lienhardt, P. (2006). A hierarchical topology-based model for handling complex indoor scenes. Computer Graphics Forum, 25(2):149-162.

Fuchs, H., Kedem, Z. M., and Naylor, B. F. (1980). On visible surface generation by a priori tree structures. SIGGRAPH Comput. Graph., 14(3):124-133.

Fujimoto, A., Tanaka, T., and Iwata, K. (1988). Arts: accelerated ray-tracing system. In Grant, C. W. and Hatfield, L., editors, Tutorial: computer graphics; image synthesis, pages 148-159. Computer Science Press.

Glassner, A. S., editor (1989). An introduction to ray tracing. Academic Press Ltd., London, UK, UK.

Goldsmith, J. and Salmon, J. (1987). Automatic creation of object hierarchies for ray tracing. IEEE Comput. Graph. Appl., 7(5):14-20.

Havran, V. (2000). Heuristic Ray Shooting Algorithms. Ph.d. thesis, Department of Computer Science and Engineering, Faculty of Electrical Engineering, Czech Technical University in Prague.

Horna, S., Damiand, G., Meneveaux, D., and Bertrand, Y. (2007). Building $3 d$ indoor scenes topology from $2 \mathrm{~d}$ architectural plans. In Conference on Computer Graphics Theory and Applications. GRAPP'2007.

Horna, S., Meneveaux, D., Damiand, G., and Bertrand, Y. (2009). Consistency constraints and $3 d$ building reconstruction. Computer-Aided Design, 41(1):13-27.

Kay, T. L. and Kajiya, J. T. (1986). Ray tracing complex scenes. SIGGRAPH Comput. Graph., 20(4):269-278.

Lienhardt, P. (1994). N-dimensional generalized combinatorial maps and cellular quasi-manifolds. International Journal on Computational Geometry and Applications, 4(3):275-324.

Luebke, D. and Georges, C. (1995). Portals and mirrors: simple, fast evaluation of potentially visible sets. In Proceedings of the 1995 symposium on Interactive $3 D$ graphics, I3D '95, pages 105-ff.
MacDonald, D. J. and Booth, K. S. (1990). Heuristics for ray tracing using space subdivision. Vis. Comput., 6(3):153-166.

Meneveaux, D., Maisel, E., and Bouatouch, K. (1998). A new partitioning method for architectural environments. Journal of Vizualisation and Computer Animation, 9(4):195-213.

Mora, F., Aveneau, L., Apostu, O., and Ghazanfarpour, D. (2012). Lazy visibility evaluation for exact soft shadows. Comput. Graph. Forum, 31(1):132-145.

Parker, S., Martin, W., Sloan, P.-P. J., Shirley, P., Smits, B., and Hansen, C. (1999). Interactive ray tracing. In Proceedings of the 1999 symposium on Interactive $3 D$ graphics, I3D '99, pages 119-126. ACM.

Plücker, J. (1865). On a new geometry of space. Philosophical Transactions of the Royal Society of London, 155:725-791.

Rubin, S. M. and Whitted, T. (1980). A 3-dimensional representation for fast rendering of complex scenes. SIGGRAPH Comput. Graph., 14(3):110-116.

Shoemake, K. (1998). Plücker coordinate tutorial. Ray Tracing News 11.

Teller, S., Fowler, C., Funkhouser, T., and Hanrahan, P. (1994). Partitioning and ordering large radiosity computations. SIGGRAPH Comput. Graph., pages 443450.

Teller, S. and Hanrahan, P. (1993). Global visibility algorithms for illumination computations. In Proceedings of the 20th annual conference on Computer graphics and interactive techniques, SIGGRAPH '93, pages 239-246, New York, NY, USA. ACM.

Teller, S. J. and Séquin, C. H. (1991). Visibility preprocessing for interactive walkthroughs. SIGGRAPH Comput. Graph., 25(4):61-70.

Wald, I., Slusallek, P., Benthin, C., and Wagner, M. (2001). Interactive rendering with coherent ray tracing. In Computer Graphics Forum, pages 153-164.

Whitted, T. (1980). An improved illumination model for shaded display. Commun. ACM, 23(6):343-349. 